\title{
Anthropometric Proportions Estimation Using recCAL in Multi-camera Environment
}

\author{
O. Kainz, D. Cymbalák, and F. Jakab
}

\begin{abstract}
In this paper, a new technique for anthropometric proportions estimation using multi-camera environment is presented. We refer to this system as a recCAL. System is represented in a form of a 3D model and as well real-world physical model, both used as a reference model/object in the size estimation process. Further anthropometric proportions of human body and face are utilized to provide an estimate of real size from series of images as captured by multi-camera system. recCAL has potential applications in variety of areas where anthropometric proportions or size of other objects may be useful.
\end{abstract}

Index Terms-Anthropometry, human proportions, object size, multi-camera.

\section{INTRODUCTION}

There are many cases when one has to determine the size of an object from the image, for example police investigators need to estimate the anthropometric features of offender directly from the image or estimate human body parameters in order to create proper 3D model. Such model may be later used for testing or in the field of clothing industry used as knowledge about body shapes tendencies of people from selected area. Estimation of size of the object in the image has been in focus of many researches. In this paper we will cover some of these and present our own approach.

We will focus primarily on estimation of selected anthropometrical features of human body. Once implemented in a real conditions as a tool in multiple camera environment, one may be able to obtain size parameters of scene relatively fast.

\section{ANTHROPOMETRIC FEATURES ESTIMATION}

Most of the cases we cover are related to body height estimation. Related to our idea is study by [1], in this research authors tried to estimate the height of human body from single uncalibrated image, however no reference towards camera viewpoint and estimation of camera parameters were provided. Localization of body landmarks was done in semi-automatic way and estimation was in this case carried out only for height. No reference length was required for the height estimation. Another research focused on height

Manuscript received January 5, 2015; revised July 23, 2015. We support research activities in Slovakia/This project is being co-financed by the European Union. Paper is the result of the Project implementation: University Science Park TECHNICOM for Innovation Applications Supported by Knowledge Technology, ITMS: 26220220182, supported by the Research \& Development Operational Programme funded by the ERDF.

Ondrej Kainz, Dávid Cymbalák, and František Jakab are with the Technical University of Kosice, Slovakia (e-mail: ondrej.kainz@tuke.sk, david.cymbalak@tuke.sk, frantisek.jakab@tuke.sk). estimation is described in [2]. In this case authors used calibrated cameras (more specifically surveillance camera). Since this was the case of calibrated camera, intrinsic parameters of camera were known. For calculation of height were used top and bottom points of person as extracted from the image itself, no anthropometric features seems to be implemented. [3] is also dealing with the height estimation, author utilized a single image containing only human face. Extraction of eyes, lip and chin enabled this estimation using statistical measurement sets and facial golden proportion (see Fig. 1). Specific process of this extraction is based on [4] and further described in [3]. Another study carried out by [5] presents again estimation of height from single camera while only requiring height, pitch angle and vanishing point of camera. Results of the testing provide good accuracy. In [6] authors focused on calibrated camera as a part of research in field of demography, where authors aimed to estimate the height of the person. Their aim was to improve age and gender classification via height estimation. Testing was carried out on 127 subjects. Authors extensively worked with anthropometric data, e.g. distance between eye centers or distance between mouth and nasion (see Fig. 1), the first is for male and female estimated to be approx. from 50 to 60 $\mathrm{mm}$ and the latter, i.e. from nasion to mouth, (b) from 45 up to $75 \mathrm{~mm}$.

Estimation of shape and pose from single image was object of the interest of research introduced in [7], authors aimed to extract 3D human model from archival images or paintings, i.e. image is uncalibrated. For initial pose estimation authors use manual selection of joints (vertices) on body. Body representation was not based directly on anthropometric model but model called SCAPE (Shape Completion and Animation of People). Body shape was derived from shading which provides strong constraints on surface shape.

\section{ANTHROPOMETRY}

Anthropometry is rather unusual field of study. Oxford dictionary [8] defines anthropometry as "the scientific study of the measurements and proportions of the human body". Word anthropometry is actually combination of two Greek words ó $v \theta \rho \omega \pi \circ \varsigma$ (man) and $\mu \varepsilon \dot{\tau} \rho \rho \nu$ (to measure). Based on [9] was anthropometry introduced in the middle of 17 th century by physician named J.S. Elsholtz for description of system of measurements with aim to describe the intrinsic relationship between body proportions and various diseases. Later on this term gained broader meaning. P. Topinard in the dawn of 20th century refers to this field as system of measurements of the human body with the goal to determine its perspective proportions. More about beginning and history of anthropometry is covered in the book by above mentioned 
author.

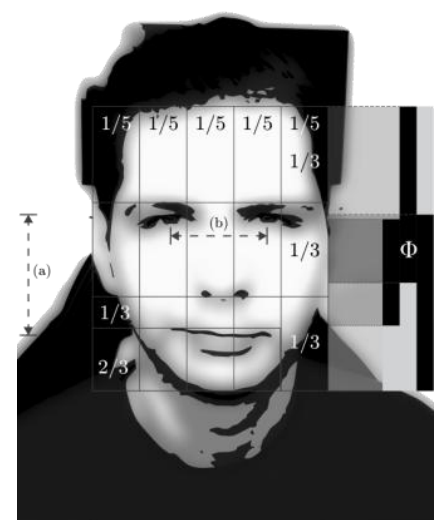

Fig. 1. Facial symmetry: a) nasion-to-mouth, b) eye-to-eye.

Nowadays anthropometry or better say its output provide utilization in many areas of life, primarily it is being used as a one of the dominant methods utilized in physical anthropology and palaeoanthropology, another use is for example in ergonomics for design of products or interiors/exteriors. There are basically two types of anthropometry presented by [10]:

- Structural (static) measurement of features in standardized (rigid) positions, movement of human body is not major concern in this type of measurement; examples are height, weight, height when sitting, body depth, elbow height et al. For measurements to be replicable on various subjects the standardized posture has to be maintained.

- Functional (dynamic) measurement of features in motion or physical activities in general, i.e. body is in various postures, e.g. arm reach, kneeling height, bent torso height et al.

Another third type of measurement introduced by [11] is:

- Newtonian measurement where the mechanical principles are applied on a human body, output of this describes segmental parameters used further in biomechanical analysis, e.g. centre of mass.

\section{BODY REPRESENTATION}

In this part we put our focus on body representation. In [12] is body represented as connection of edges joined by vertices (axes of rotation). These links are merely abstractions of constant length that allow angular displacement, none of which is truly edge-centered. More about link body system and its dynamic properties is described in publication by just mentioned authors. [13] used model of human body consisted from vertices, edges and faces as a foundation for human model. Same authors also developed a system called SASS (Spreadsheet Anthropometry Scaling System) for generating the human figure based on population data as an input.

Vertex in our case is connection between bones, i.e. synovial type of joins and diarthrosis, well known of these is e.g. knee or elbow (in Fig. 2 see rk, lk and re, le, respectively). All the joints that are synovial are freely moveable, however each type has some level of movability. Most of the vertices are of type diarthrosis. In Fig. 2 is depicted human body model consisted of edges end vertices.

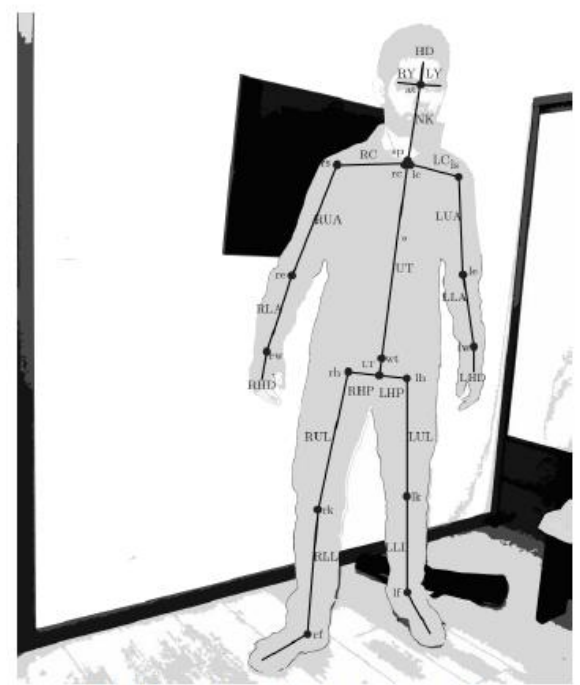

Fig. 2. Skeleton human body model: vertices and edges.

TABLE I: DESCRIPTION OF EDGES FOR FIG. 2 [14]

\begin{tabular}{ll|ll}
\hline \hline Edge & Name & Edge & Name \\
\hline HD & HeaD & RHD & Right HanD \\
RY & Right eYe & LHD & Left HanD \\
LY & Left eYe & LT & Lower Torso \\
NK & NecK & RHP & Right HiP \\
UT & Upper Torso & LHP & Left HiP \\
RC & Right Clavicle & RUL & Right Upper Leg \\
LC & Left Clavicle & LUL & Left Upper Leg \\
RUA & Right Upper Arm & RLL & Right Lower Leg \\
LUA & Left Upper Arm & LLL & Left Lower Leg \\
RLA & Right Lower Arm & RF & Right Foot \\
LLA & Left Lower Arm & LF & Left Foot \\
\hline \hline
\end{tabular}

TABLE II: DESCRIPTION OF VERTICES FOR FIG. 2 [14]

\begin{tabular}{ll|ll}
\hline \hline Vertex & Name & Vertex & Name \\
\hline at & atlanto occipital & ra & right ankle \\
sp & solar plexus & rc & right clavicle \\
la & left ankle & re & right elbow \\
lc & left clavicle & rh & right hip \\
le & left elbow & rk & right knee \\
lh & left hip & rs & right shoulder \\
lk & left knee & rw & right wrist \\
ls & left shoulder & wt & waist \\
lw & left wrist & & \\
\hline \hline
\end{tabular}

\section{RECCAL DESIGN}

recCAL is basically rectangular cuboid for calibration of camera with the aim to estimate the unknown size of objects in the image. Basis presumption is that this device is present in the image. Further we utilize the facial anthropometric measures and also anthropometric measures of human body. Illustration of recCAL is depicted in Fig. 3. recCAL is 3D model that is loaded into the image based on extraction of feature points from the real world device, once located mapping is of 3D model into the image is carried out. When located, parameters of image and estimation of camera parameters are enabled. Carried out next is detection of human face based on feature extraction presented in [15]. 
Then vertices are located following the skeleton model of human body, this is based on extreme point detection, see analogous approach in [2]. Final result is the estimation of anthropometric proportions and partial pose estimation. These data may then serve as an input for metric calculation of multiple camera sources.

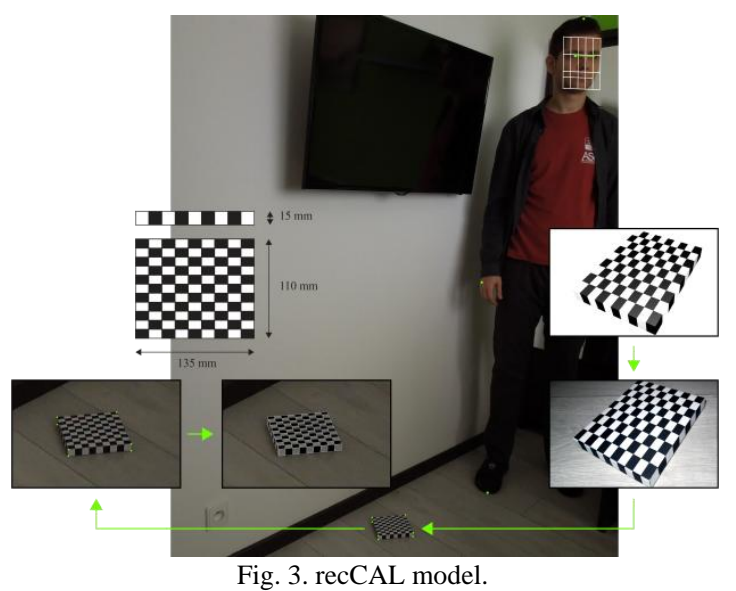

\section{Building OF SKeleton Body Model From Multiple CAMERAS AND RECCAL}

Previous approaches consider primarily usage of static photos for calculation of the proportions of vertices. Our revolutional approach bring the advantages of using video for calculating the vertices by dividing the computing capacity to multiple camera sources via searching the diffractive points of human body. These points are evaluated by optical flow algorithm during the observation of walking or moving habits using computer visions techniques (Fig. 4).

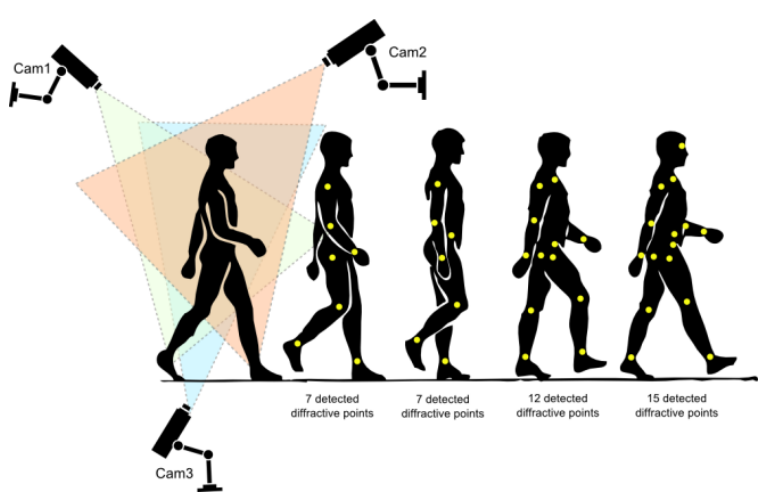

Fig. 4. Detecting the diffractive points from multiple angles.

Once diffractive points are detected, the process of calculation the distance between these points is carried out, this is done in order to get value of chosen vertices in skeleton model. These values are initially set from the first camera view and also updated by next cameras view to the final model related to the reference recCAL (Fig. 5).

The result of overall calculations is creation of vector of chosen vertices values:

$$
H_{n}^{t}=\left[H D_{n}, R Y_{n}, L Y_{n}, U T_{n}, L U A_{n}, R L A_{n}, L U L_{n}, R L L_{n}\right]
$$

This vector is used in real time for track and prediction of movement of specific human. This solution was tested in experimental laboratory environment by using the software vMix system and also the cloned HDMI input cards and scripts for evaluation of the skeleton model vector (Fig. 6).
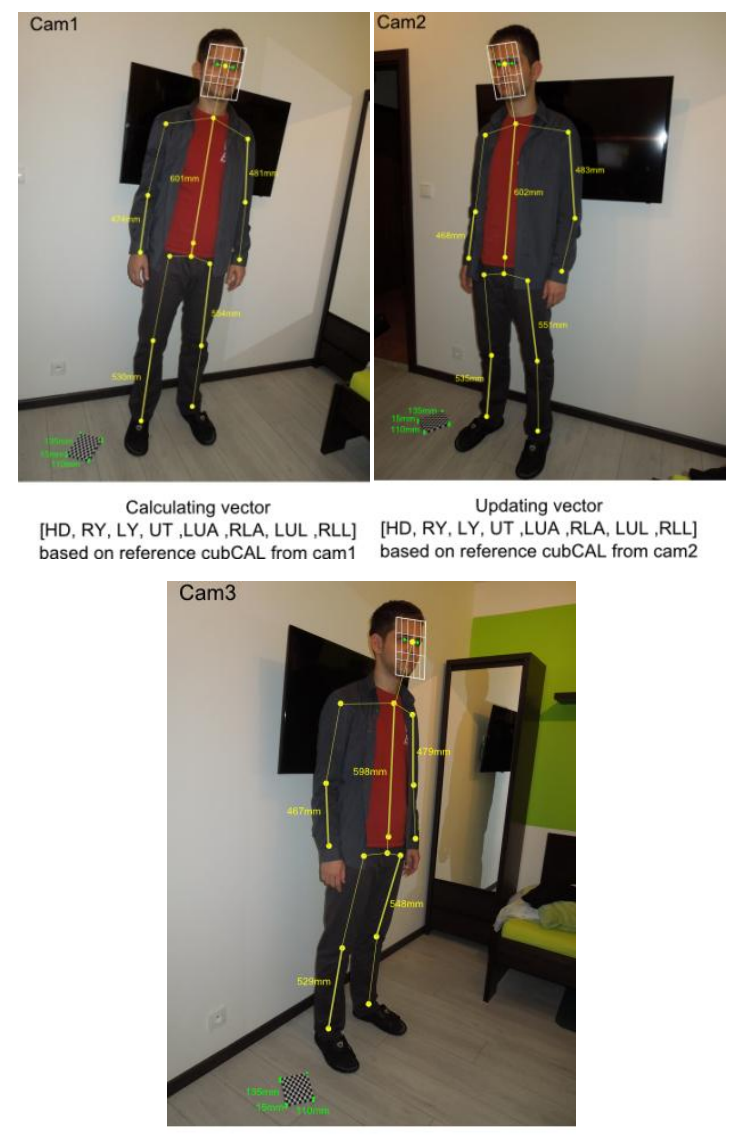

Updating and finalising vector

[HD, RY, LY, UT ,LUA , RLA, LUL, RLL]

based on reference cubCAL from cam3

Fig. 5. Calculating vertices and updating skeleton model from multiple angles.

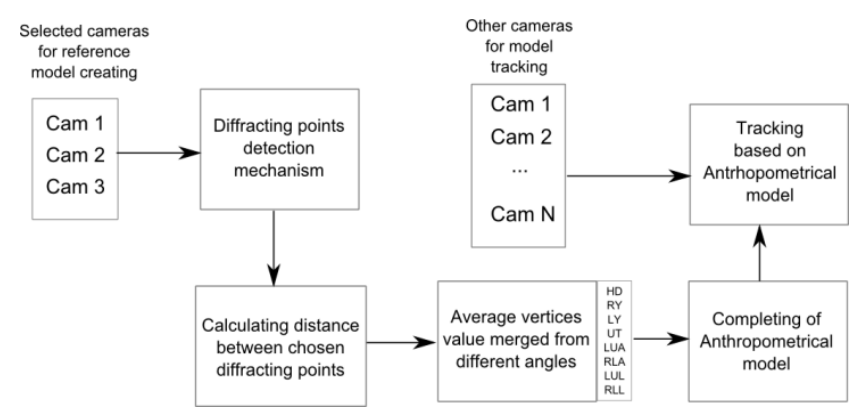

Fig. 6. Process of creation of skeleton model with reusing model for tracking the human through different video sources.

\section{CONCLUSION}

recCAL prototype system consists from device and $3 \mathrm{D}$ model, both of them are introduced as an aid in for measurement the object size in the scene using multi-camera approach. Proposed solution is able to estimate anthropometric proportions due to homogeneous proportions of human body. In this study we were able to partially estimate certain of these anthropometric proportions following skeleton model of human body and facial proportions. Knowing recCAL parameters enabled us to estimate parameters from the image and map its 3D model to scene itself. In future we plan to extend the number of vertices in skeleton model and thus enhance the accuracy of body parameters estimation, potential is to utilize system for calculating the parameters of the whole scene. 


\section{REFERENCES}

[1] C. H. B. Abdelkader and Y. Yacoob, "Statistical body height estimation from a single image. FG '08," in Proc. the 8th IEEE International Conference on Automatic Face and Gesture Recognition, pp. 1-7, 2008.

[2] I. Kispál and E. Jeges. (2014). Human height estimation using calibrated camera. [Online]. Available: http://webmail.mit.bme.hu/eng/research/search/downloads/ibar/Huma n_height_estimation_using_a_calibrated_camera.pdf.

[3] Y. P. Guan, "Unsupervised human height estimation from a single image," Journal of Biomedical Science and Engineering, pp. 425-430, 2009.

[4] Y. P. Guan, "Robust eye detection from facial image based on multi-cue facial information," IEEE International Conference on Control and Automation, pp. 1775-1778, 2007.

[5] M. K. Momeni, C. H. S. Diamantas, F. Ruggiero, and B. Siciliano, "Height estimation from a single camera view," International Conference on Computer Vision Theory and Applications, pp. 358-364, 2012.

[6] A. C. Gallagher, A. C. Blose, and T. Chen, "Jointly estimating demographics and height with a calibrated camera." in Proc. IEEE 12th International Conference on Computer Vision, 2009.

[7] Y. P. Guan, A. Weiss, A. O. Balan, and M. J. Black, "Estimating human shape and pose from a single image," in Proc. IEEE 12th International Conference on Computer Vision (ICCV), pp. 1381-1388, 2009.

[8] A. Stevenson, Oxford Dictionary of English, Oxford: Oxford University Press, 2010.

[9] F. Spencer, History of Physical Anthropology: An Encyclopedia, vol. 1, New York: Garland, 1997.

[10] M. A. Friend and J. P. Kohn, Fundamentals of Occupational Safety and Health, Plymouth: Government Institutes, 2010.

[11] D. Grieve and S. Pheasant, "Biomechanics," The Body at Work: Biological Ergonomics, pp. 71-200, 2010.

[12] W. T. Dempster, "The anthropometry of body actions," Annals of the New York Academy of Sciences, pp. 559-585, 2006.

[13] M. R. Grosso, R. D. Quach, E. Otani, J. Zhao, S. Wei, P.-H. Ho, J. Lu, and N. J. Badler, "Anthropometry for computer graphics human figures," State-of-the-Art in Computer Animation: Proceedings of Computer Animation '89, pp. 83-96, 1989.

[14] C. Barrón, and I. A. Kakadiaris, "On the improvement of anthropometry and pose estimation from a single uncalibrated image." Journal Machine Vision and Applications - Special Issue: Human Modeling, Analysis, and Synthesis, pp. 229-236, 2003.

[15] K. Sridharan and V. Govindaraju, "A sampling based approach to facial feature extraction," Fourth IEEE Workshop on Automatic Identification Advanced Technologies, pp. 51-56. 2005.

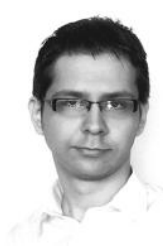

Ondrej Kainz was born in 1988. He received his MSc in applied informatics from the Technical university in Kosice, Slovakia in 2013. Since the very same year he is a $\mathrm{PhD}$ student at the Department of Computers and Informatics of the Faculty of Electrical Engineering and Informatics at the Technical University of Kosice. His scientific research interests include computer vision, e-learning, human-computer interfaces, computer graphics, computer networks, biological engineering and body area network.

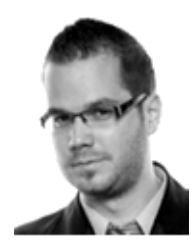

Dávid Cymbalák was born in 1987. In 2011 he graduated with (MSc) at the Department of Computers and Informatics of the Faculty of Electrical Engineering and Informatics at Technical University in Kosice. Since 2011 he is a PhD student at the Department of Computers and Informatics. His scientific research is focused on streaming technologies, e-learning solutions or object tracking methods. In addition, he also investigates questions related with the optimization of mobile access to multimedia sources.

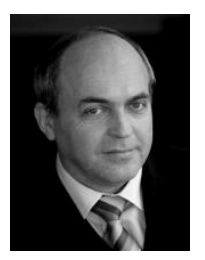

František Jakab was born in 1959. He received his MSc in system engineering from St. Petersburg Electro Technical University, Russia, in 1984, and PhD degree from Technical university of Kosice in Slovakia, in 2005. $\mathrm{He}$ as a associated professor since 2008 . He has extensive experience in networking and utilization of ICT in education (more then 30 years teaching experience) where he established well known research centre Computer Networks Laboratory (www.cnl.sk, in 1995). He has been an coordinator of several large international projects financed by EC, coordinator of national wide ICT projects and research grants. He act as an expert in areas: projecting of computer networks, new form of multimedia based communication (videoconferences, IP streaming, IPTV, LMS). Published more than 150 papers, 5 books (author, co-author.); Chair of many International Symposiums and conferences, editor conference Proceedings. Since 1999 involved into Cisco Networking academy program in Slovakia as an instructor (CCAI certification) and since 2001 in position of coordinator of the Program in Slovakia (network of 65 universities and secondary schools), 2008-2014 Regional Lead for Russia, Ukraine and CIS. He is a head of the Application Section of the Communication Technology Forum Association in Slovakia, head of Committee on business - academic cooperation, American Chambers of Comers in Slovakia and general manager of University Centre for Innovation, Technology Transfer and Intelectual Property Protection at Technical university of Kosice. He was awarded as a "IT person of the year 2006" in Slovakia. 